\title{
Binary precession solutions based on synchronized field couplings
}

\author{
Jamahl Peavey \\ Department of Science, Arlington School, 115-Mill Street, Belmont, MA 02478, U.S.A \\ arlingtonpeavey@yahoo.com
}

\begin{abstract}
A classical wave function is presented as an interface between a gravitational and an electromagnetic field. Binary star precessions are calculated as a best test for the most important interface in physics. Among the ten most difficult systems tested are DI Herculis, V541 Cygni and AS Camelopardalis. These systems have observed precessions which are not consistent with theoretical predictions. The precession results based on the classical wave function prove there is a fundamental relationship between Newtonian mechanics, general relativity and quantum mechanics.
\end{abstract}

Keywords: Wave functions, binary stars, double slit experiment, precession, dark matter.

Introduction

The classical wave function or harmonic space is constructed from structures consistent with the wave function $\psi(x, t)$, the fine structure constant $\alpha$, the principle quantum number $\eta$ and electron orbital dynamics. These four structures are associated with atomic systems. A generalized equivalence for each structure is produced to construct a classical wave function that is consistent with Newtonian mechanics and general relativity. The classical wave function synchronizes a binary star system's violent gravitational and electromagnetic field, thereby producing what is detected as precession or apsidal motion.

\section{Method}

Synchronization exists between harmonic systems coupled with a varying space. Pendulums with varying frequencies will remain varied on a fixed platform. If the platform is allowed to vibrate horizontally, the pendulums will obtain the same frequency or synchronize. If a gravitational field and an electromagnetic field are treated as pendulums on a vibrating platform, then both fields will synchronize or move in phase.

\section{The generalized orbit}

Newton's universal law of gravitation must be true for three classical constraints on space: Mass cannot exist at a point; Resistance to mass superposition is volume displacement; a system boundary must be established to apply the laws of conservation. Consider, placing a system boundary on a spherical surface. A point on the surface produces a gravitational acceleration independent of the point's mass. This violates the first constraint and giving the point volume, violates the second constraint unless volume is displaced. If volume is displaced, then the system boundary cannot be maintained at the spherical surface. The only system boundary that can support these spatial constraints and Newton's universal law of gravitation is an orbit. The three general functions for orbits: Orbits delocalize three dimensional objects to prevent singularities; orbits represent objects harmonically extended and in phase with a sinusoidal wave; orbits regionalize space into an interior space (enclosed by the orbit) and into an exterior space (outside the orbit). Unlike, the electron orbit's standing waves, these sinusoidal waves are coplanar with the orbit. The coplanar configuration means that the object's path is transitionally continuous and deterministic.

\section{The generalized principle quantum number}

Hydrogen spectral lines are described by eqn. 1 . The principle quantum numbers $n_{i}$ and $n_{f}$ support in phase field harmonics, not continuous motion. A generalized principle quantum number must support in phase field harmonics and the object's continuous motion. The generalized structure is deduced by analyzing the hydrogen spectrum.

$$
1 / \lambda=R_{H}\left(1 / \eta_{i}^{2}-1 / \eta_{f}^{2}\right)
$$

The spectral pattern appears to support natural numbers and a converging geometric series $g=1 /(1-k)$ such that $\left(1 / g_{i}^{2}-1 / g_{f}^{2}\right)$ is true. Observation shows that each principle quantum number is a series of converging wavelengths.

\section{The classical wave function}

The classical wave function is an interface between an interior and an exterior space. Each space has a spin characteristic which supports the in phase condition for a field. The spin characteristic is precession. The interior spatial spin which produces an in phase interior is described by eqn. 2 and theta inverse function is described by eqn. 3 . The in phase interior space has a hydrogen spectral configuration.

$$
\operatorname{Tan}(\theta)=\frac{1}{\left[1-\left(1 / g^{2}\right)\right]}
$$




$$
\theta^{-1}=\operatorname{Tan}\left(\frac{1}{\left[1-\left(1 / g^{2}\right)\right]}\right)
$$

The exterior spatial spin which produces an in phase exterior is described by eqn. 4 and theta inverse function is described by eqn. 5. The term $g_{o}$ means the geometric series is on the exterior space.

$$
\begin{aligned}
& \operatorname{Cos}(\theta)=g_{o}-1 \\
& \theta^{-1}=\operatorname{Cos}\left(g_{o}-1\right)
\end{aligned}
$$

The precession is $\Delta \theta=\left(90^{\circ}-\theta\right)$ for eqn. 2 and 4 . Eqn. 2 is confirmed by calculating the deflection of light passing the sun and eqn. 4 is confirmed by calculating Mercury's precession. The system precession or interface between the interior and exterior spatial spin is described by eqn. 6 and the inverse is described by eqn. 7. The amplitude $A$, shape factor $(1-e)$ and size factor $(1+0.25 \varepsilon)$ completes the most general interface or classical wave function. Eqn. 6 and 7 are the frequency forms for the interface. Eqn. 7 describes the inverse function for the frequency form.

The classical wave functions \& precession relationship

$$
\Delta \omega(R)=\operatorname{Sin}^{-1}\left(\left[g_{o}-1\right] / A\left[1-\left(1 / g^{2}\right)\right]\right)(1-e)(1+0.25 \varepsilon) / \text { cycle }
$$

$\Delta \omega^{-1}(R)=A \operatorname{Sin}\left(\left[g_{o}-1\right] /\left[1-\left(1 / g^{2}\right)\right]\right)(1-e)(1+0.25 \varepsilon) /$ cycle

Let $R$ represent the initial position. If $R=R_{p}$ then the system is expanding or $\varepsilon=\varepsilon_{+}=\left(R_{a}-R_{p}\right) / R_{p}$. If $R=R_{a}$ then the system is contracting or $\varepsilon=\varepsilon_{-}=\left(R_{p}-R_{a}\right) / R_{a}$. The perihelion distance is $R_{p}$ or the closest orbital position, and the aphelion distance $R_{a}$ is the farthest orbital position. The eccentricity or $e$ is the orbital shape and a cycle is one year. The amplitude $A$ is conditionally based on the coupling position. Eqn. 6 and 7 are confirmed by calculating binary star precessions.

The gravitational couplings

The classical wave function's geometry describes precession and coupled to that geometry are forces. The common ratio $k$ for a converging geometric series has the form $(v / c)^{2}$. The object's velocity $v$ to light speed $c$ is a fundamental ratio in special and general relativity. An interaction's strength or coupling is measured by this ratio. The gravitational field has three coupling configurations. Let $M_{p}$ and $M_{s}$ represent the primary and secondary mass respectively, while $G$ is the universal gravitational constant, and $R$ is the distance from the source.

1. Escape velocity's squared coupling form $\beta_{e s}^{2}=\frac{2 G M_{p}}{c^{2} R}$ is the equivalence between the secondary mass kinetic energy and the gravitational potential energy between both masses.

2. Orbital velocity's squared coupling form $\beta_{o}^{2}=\frac{G M_{p}}{c^{2} R}$ is the equivalence between the secondary mass centrifugal force and the gravitational force between both masses. Detached binary systems have this coupling.

3. Collapse velocity's squared coupling form $\beta_{c o l}^{2}=\frac{2 G\left(M_{p}+M_{s}\right)}{c^{2} R}$ is the equivalence between both masses kinetic energy and the gravitational potential energy between total mass. Contact and semi-detached binary systems have this coupling.

Interior configuration test

The sun acts as an orbit such that $R$ is the sun's radius and $M_{p}$ is the sun's mass. The interior space has escape velocity coupling. Calculate $g=1 /\left(1-\beta_{\text {es }}^{2}\right)$ and substitute it into Eqn. 2. The precession is $\Delta \theta=\left(90^{\circ}-\theta\right)=1.748$ arc sec. Starlight is deflected 1.75 arc sec as it passes the sun. This value is predicted by general relativity.

\section{Exterior configuration test}

The sun acts as an orbit such that $R$ is the sun's radius and $M_{p}$ is the sun's mass. Any object within this region experiences orbital velocity coupling just outside the sun. Calculate $g_{o}=1 /\left(1-\beta_{o}^{2}\right)$ and substitute it into
Research article

CIndian Society for Education and Environment (iSee)
Jamahl Peavey Indian J.Sci.Technol. 
eqn. 4. The precession is $\Delta \theta=\left(90^{\circ}-\theta\right)=0.437$ arc sec/cycle. This means there are 43.7 arc sec/100 years. Mercury's observed precession is $43.1 \mathrm{arc} \mathrm{sec} / 100$ years because it orbits the sun at $R_{p}=45.9 \times 10^{6} \mathrm{~km}$. The precession drop at perihelion is $\Delta \theta_{\text {drop }}=\left(90^{\circ}-\theta_{p}\right)=0.660$ arc sec $/ 100$ years. Mercury's observed precession is approximately equal to $\Delta \theta-\Delta \theta_{\text {drop }}$. This value is also predicted by general relativity. The precession drop proves Mercury's orbit is extending the sun's interior space and is reducing the exterior space. Both tests confirm the first, second and third function for orbits. These configuration tests prove the classical wave function's structure is consistent with Newtonian mechanics, general relativity and quantum mechanical structures.

\section{Electromagnetic coupling}

The fine structure constant $\alpha=1 / 137$ measures the strength for the electromagnetic interaction or electromagnetic coupling. The fine structure constant has more than one accepted form. The original form is $\alpha=v_{e} / c$ such that $v_{e}$ is the electron's hydrogen ground state velocity. The squared coupling form $\alpha^{2}$ is the common ratio $k$ for a converging geometric series.

\section{Gravitation \& electromagnetic interface:}

If field $A$ is coupled to the interior space and field $B$ is coupled to the exterior space, then the classical wave function synchronizes both fields as a classical unification. If $\alpha^{2}$ is coupled to one space, then $\beta^{2}$ is coupled to the other space. This conditional statement produces the four possible configurations described by eqn. 8, 9, 10 and 11 . The amplitude magnitude is based on coupling location.

Gravitation \& electromagnetic interface conditions:

If $g_{o}=1 /\left(1-\beta^{2}\right)$ and $g=1 /\left(1-\alpha^{2}\right)$ then $A=1$ for a

variation 1 system to exist.

$$
\Delta \omega(R)=\operatorname{Sin}^{-1}\left(\left[g_{o}-1\right] / A\left[1-\left(1 / g^{2}\right)\right]\right)(1-e)(1+0.25 \varepsilon) / \text { cycle }
$$

If $g_{o}=1 /\left(1-\alpha^{2}\right)$ and $g=1 /\left(1-\beta^{2}\right)$ then $A \geq 10$ for a
$\Delta \omega(R)=\operatorname{Sin}^{-1}\left(\left[g_{o}-1\right] / A\left[1-\left(1 / g^{2}\right)\right]\right)(1-e)(1+0.25 \varepsilon) /$ cycle

If $g_{o}=1 /\left(1-\beta^{2}\right)$ and $g=1 /\left(1-\alpha^{2}\right)$ then $A \geq 10$ for a

Variation 3 system to exist. Eqn. 9 and 10 are inverses because field couplings have changed regions.

$\Delta \omega^{-1}(R)=A \operatorname{Sin}\left(\left[g_{o}-1\right] /\left[1-\left(1 / g^{2}\right)\right]\right)(1-e)(1+0.25 \varepsilon) /$ cycle

If $g_{o}=1 /\left(1-\alpha^{2}\right)$ and $g=1 /\left(1-\beta^{2}\right)$ then $A=1$ for a

Variation 4 system to exist. Eqn. 8 and 11 are inverses because field couplings have changed regions.

$\Delta \omega^{-1}(R)=A \operatorname{Sin}\left(\left[g_{o}-1\right] /\left[1-\left(1 / g^{2}\right)\right]\right)(1-e)(1+0.25 \varepsilon) /$ cycle

The $A_{\text {min }}=10^{n}, A_{\text {max }}=A_{\text {min }}(1+0.25 \varepsilon)$ and $n$ supports whole numbers. The V1 and V4 systems exist at $n=0$ while the $\mathrm{V} 2$ and $\mathrm{V} 3$ systems exist over a $n \geq 1$ range. Wave function interference has statistical form, so precessions must also have statistical form. Average and normalized

$\Delta \omega(R)_{\text {avg }}=\left[\Delta \omega(R)_{\min }+\Delta \omega(R)_{\max }\right] / 2$

precessions

$\Delta \omega(R)_{\text {norm }}=\Delta \omega(R)_{\min } \pm 0.10 \Delta \omega(R)_{\text {min }}$ are given respectively. The average precession is $\mathrm{V} 2$ and $\mathrm{V} 3$ based. The normalized precession is V1 and V4 based.

\section{Results and discussion}

Table 1 referenced data contains approximate values. The terms $M$ and $R$ represent the sun's mass and radius respectively. The projected semi-major axis $a_{o}$ is always taken from the primary mass orbit. The PSR B1534+12, PSR B2127+11C, PSR J1518+4904 and PSR $B 2303+46$ systems data is from (Nice et al., 1996). The PSR B1913+16 system data is from Johnston (2004). The PSR J1756-2251 system data is from (Burgay et al., 2005). The PSR JO737-3039A system data is from (Burgay et al., 2006). The V541 Cygni system data is from (Claud \& Sandberg, 1998). The DI Herculis system

variation 2 system to exist.

Research article

CIndian Society for Education and Environment (iSee)
Jamahl Peavey Indian J.Sci.Technol. 
Table 1. Observed precessions.

\begin{tabular}{|c|c|c|c|c|c|c|}
\hline BINARY STARS & Form & $M_{p}$ & $M_{p}+M_{s}$ & $e$ & $a_{o}$ & $\Delta \omega(R)_{\text {observed }}$ \\
\hline PSR B1913+16 & Contact & $1.441 M$ & $2.828 M$ & 0.617 & $2.80 R$ & $4.2^{\circ} / y r$ \\
\hline PSR B1534+12 & Semi-Detached & & $2.678 M$ & 0.274 & $3.73 R$ & $1.756^{\circ} / y r$ \\
\hline PSR B2127+11C & Semi-Detached & & $2.712 M$ & 0.681 & $2.52 R$ & $4.457^{\circ} / y r$ \\
\hline PSR J1756-2251 & Semi-Detached & & $2.574 M$ & 0.1805 & $2.756 R$ & $2.585^{\circ} / y r$ \\
\hline PSR J0737-3039A & Contact & $1.338 M$ & $2.587 M$ & 0.0877 & $1.415 R$ & $16.89^{\circ} / y r$ \\
\hline PSR J1518+4904 & Contact & & $2.62 M$ & 0.249 & $20.04 R$ & $0.0111^{\circ} / y r$ \\
\hline PSR B2303+46 & Contact & & $2.60 M$ & 0.658 & $32.69 R$ & $0.010^{\circ} / \mathrm{yr}^{\prime}$ \\
\hline V541 Cygni & Detached & $2.24 M$ & $4.48 M$ & 0.479 & $1.88 R$ & $0.60^{\circ} / 100 \mathrm{yr}$ \\
\hline DI Herculis & Detached & $5.185 M$ & $8.719 M$ & 0.489 & $2.68 R$ & $1^{\circ} / 100 \mathrm{yr}$ \\
\hline AS Camelopardalis & Detached & $3.3 M$ & $5.8 M$ & 0.1695 & $2.57 R$ & $15 \pm 5.0^{\circ} / 100 \mathrm{yr}$ \\
\hline
\end{tabular}

data is from (Claret, 1998). The AS Camelopardalis system data is from (Guinan et al., 1991).

Note: The V2 system has maximum precession at minimum amplitude and the reverse is true. The V3 and V4 systems minimum precessions refer to $\Delta \omega^{-1}(R)_{\text {min }}$ while the V3 system's maximum precession refers to $\Delta \omega^{-1}(R)_{\max }$. The $\mathrm{V} 1$ semi-detached systems have the same structure as the V1 contact system (Table 2). The V1 semi-detached systems logically started as contact systems and separated. The separation caused normalization about the contact precession. The statistics associated with normalized and average precession superposition are similar to the double slit experiment's statistics for wave function interference. The standard deviation $\sigma= \pm 0.10 \Delta \omega(R)_{\min }$ is not uncertainty but a fundamental feature associated with harmonic space. Multiple outcomes are associated with one condition and multiple measurements simply reproduce the statistical distribution of acceptable outcomes. The PSR J07373039A and PSR J1518+4904 systems have measurable average precessions. PSR B2303+46's minimum precession is measurable. The V541 Cygni and DI Herculis systems have measureable minimum and maximum precessions. Therefore, both precessions are valid. Measurement cannot determine the actual state because celestial measurement methods cannot stop a binary system's precession whereby collapsing its associated wave function. Subatomic precessions are measurement based because photons can stop precession, collapse the wave function and identify the system's actual state at that moment. The AS Camelopardalis system's contracted state may indicate reversibility for all systems.

\section{Conclusion}

The classical wave function is the most general form for $\psi(x, t)$ and the binary stars tested prove the structure and its statistical features are real. The classical wave function describes the motion of two stars. Therefore, its features must exist within galaxies and galaxy clusters. Dark matter is the galactic feature for $A \geq 10$. Dark to normal matter ratio is supported by $A_{\text {min }}=10^{n}$ and $A_{\max }=A_{\text {min }}(1+0.25 \varepsilon)$ which means dark matter is harmonic space intensity. Dark energy and dark flow are the galactic equivalence for the classical wave function's expansive and contracted states respectively. These dynamic states evolve without energy because wave functions do not carry energy. Galaxy clusters and stars within galaxies are entangled because binary stars are described by a wave function. The gravitational and electromagnetic couplings are symmetric within the classical wave function. Therefore, gravity is an ordinary force and the classical wave function description of a harmonic space is Einstein's space-time. The symmetry also proves the electron is a particle moving through this harmonic space. 
Table 2. Calculated precessions.

\begin{tabular}{|c|c|c|c|c|c|c|}
\hline BINARY STARS & Type & $n$ & $\begin{array}{l}R_{p}=a_{o}(1-e) \\
R_{a}=a_{o}(1+e)\end{array}$ & $\varepsilon$ & $\begin{array}{l}A_{\min } \\
A_{\max }\end{array}$ & $\begin{array}{l}\Delta \omega(R)_{\min } \\
\Delta \omega(R)_{\max }\end{array}$ \\
\hline PSR B1913+16 & $\mathrm{V} 1$ & 0 & $\begin{array}{c}R_{p}=746,600 \mathrm{~km} \\
R_{a}=3,153,600 \mathrm{~km}\end{array}$ & 3.223 & 1 & $4.133^{\circ} / y r$ \\
\hline PSR B1534+12 & $\mathrm{V} 1$ & 0 & $\begin{array}{l}R_{p}=1,884,754 \mathrm{~km} \\
R_{a}=3,307,406 \mathrm{~km}\end{array}$ & 0.755 & 1 & $1.944 \pm 0.1944^{\circ} / y r$ \\
\hline PSR B2127+11C & $\mathrm{V} 1$ & 0 & $\begin{array}{c}R_{p}=559,500 \mathrm{~km} \\
R_{a}=2,948,339 \mathrm{~km}\end{array}$ & 4.269 & 1 & $5.081 \pm 0.5081^{\circ} / y r$ \\
\hline PSR J1756-2251 & $\mathrm{V} 1$ & 0 & $\begin{array}{l}R_{p}=1,571,945 \mathrm{~km} \\
R_{a}=2,264,406 \mathrm{~km}\end{array}$ & 0.4405 & 1 & $2.362 \pm 0.2362^{\circ} / y r$ \\
\hline PSR J0737-3039A & V2 & 1 & $\begin{array}{c}R_{p}=898,469 \mathrm{~km} \\
R_{a}=1,071,210 \mathrm{~km}\end{array}$ & 0.1925 & $\begin{array}{c}10 \\
10.481\end{array}$ & $\begin{array}{l}17.49^{\circ} / y r \\
16.66^{\circ} / y r\end{array}$ \\
\hline PSR J1518+4904 & V3 & 2 & $\begin{array}{l}R_{p}=10,474,828 \mathrm{~km} \\
R_{a}=17,420,852 \mathrm{~km}\end{array}$ & 0.6631 & $\begin{array}{c}100 \\
116.57\end{array}$ & $\begin{array}{l}0.01056^{\circ} / y r \\
0.01232^{\circ} / y r\end{array}$ \\
\hline PSR B2303+46 & V3 & 2 & $\begin{array}{c}R_{p}=7,781,266 \mathrm{~km} \\
R_{a}=37,723,214 \mathrm{~km}\end{array}$ & 3.847 & $\begin{array}{c}100 \\
196.19\end{array}$ & $\begin{array}{l}0.0108^{\circ} / y r \\
0.0212^{\circ} / y r\end{array}$ \\
\hline V541 Cygni & V3 & 1 & $\begin{array}{c}R_{p}=681,718 \mathrm{~km} \\
R_{a}=1,935,242 \mathrm{~km}\end{array}$ & 1.838 & $\begin{array}{c}10 \\
14.596\end{array}$ & $\begin{array}{l}0.603^{\circ} / 100 y r \\
0.881^{\circ} / 100 y r\end{array}$ \\
\hline DI Herculis & V3 & 1 & $\begin{array}{c}R_{p}=953,158 \mathrm{~km} \\
R_{a}=2,777,402 \mathrm{~km}\end{array}$ & 1.913 & $\begin{array}{c}10 \\
14.782\end{array}$ & $\begin{array}{l}0.992^{\circ} / 100 y r \\
1.46^{\circ} / 100 y r\end{array}$ \\
\hline AS Camelopardalis & V4 & 0 & $\begin{array}{l}R_{p}=1,485,532 \mathrm{~km} \\
R_{a}=2,091,908 \mathrm{~km}\end{array}$ & -0.289 & 1 & $15.3^{\circ} / 100 y r$ \\
\hline
\end{tabular}

\section{References}

1. Burgay M, Camilo F, D'Amico N, Faulkner AJ, Hobbs G, Kramer M, Lorimer DR, Lyne AG, Manchester RN, McLaughlin MA, Possenti A and Stairs IH (2005) PSR J1756 $\overline{\mathrm{A}}$ 2251: a new relativistic double neutron star system. A.P.J. 618, L119-L122.

2. Burgay $M$, Camilo $F$, D'Amico N, Ferdman RD, Freire PCC, Hobbs GB, Kramer M, Lorime DR, Lyne AG, Manchester RN, McLaughlin MA, Possenti A, Reynolds JE, Sarkissian JM and Stairs IH (2006) Relativity from timing the double pulsar. Science. 314 (5796), 97-102.

3. Claret A (1998) Some notes on the relativistic apsidal motion of DI Herculis. Astronomy \& Astrophysics. 330, 533-540.

4. Claud H. Sandberg Lacy (1998) Absolute dimensions and masses of $\mathrm{V} 541 \mathrm{CYGNI}$ and the general theory of relativity. Astronomical. J. 115, 801-808.

5. Maloney FP, Guinan EF and Mukherjee J (1991) Eclipsing binary stars as tests of gravity theories: new solutions for AS Camelopardalis. Astronomical J. 102, 256-261.

6. Johnston R (2004) Johnston's archive. Binary pulsar PSR B1913+16. Retrieved (May 01, 2006) from http://www. johnstonsarchive.net/ relativity/ binpular.html

7. Nice DJ, Taylor JH and Sayer RW (1996) PSR J1518+4904: A mildly relativistic binary pulsar system Astrophys.J.Lett. 446, L87-L90. 